\title{
Antimicrobial susceptibilities of Proteus mirabilis: a longitudinal nationwide study from the Taiwan surveillance of antimicrobial resistance (TSAR) program
}

Jann-Tay Wang ${ }^{1,2}$, Pei-Chen Chen², Shan-Chwen Chang ${ }^{1,3}$, Yih-Ru Shiau², Hui-Ying Wang ${ }^{2}$, Jui-Fen Lai I-Wen Huang ${ }^{2}$, Mei-Chen Tan², Tsai-Ling Yang Lauderdale ${ }^{2^{*}}$ and TSAR Hospitals

\begin{abstract}
Background: Longitudinal nationwide data on antimicrobial susceptibility in Proteus mirabilis from different sources are rare. The effects of the revised Clinical and Laboratory Standards Institute (CLSI) $\beta$-lactam breakpoints on susceptibility rates and on detecting extended-spectrum $\beta$-lactamase (ESBL) and AmpC $\beta$-lactamase-producers in this species are also seldom evaluated. The present study analyzed data from the Taiwan Surveillance of Antimicrobial Resistance program to address these issues.

Methods: Isolates were collected biennially between 2002 and 2012 from 25 to 28 hospitals in Taiwan. Minimum inhibitory concentrations (MIC) were determined by reference broth microdilution method. All isolates with aztreonam, ceftazidime, or cefotaxime MIC $\geq 2 \mathrm{mg} / \mathrm{L}$ were checked for the presence of ESBL by CLSI confirmatory test and subjected to ESBL and AmpC $\beta$-lactamases gene detection by PCR. Univariate and multivariate analyses were performed.

Results: Between 2002 and 2012, a total of 1157 P. mirabilis were studied. Susceptibility to cefotaxime, ceftazidime, and ciprofloxacin decreased significantly during the past decade, from $92.6 \%$ to $81.7 \%, 100 \%$ to $95.2 \%$, and $80.1 \%$ to $53.8 \%$, respectively $(P<0.01)$. The revised CLSI breakpoints had significant impact on susceptibility to cefazolin (2009 vs. current breakpoints, $71.9 \%$ vs. $0.9 \%$ ) and imipenem (99.8\% vs. 55.1\%) ( $P<0.001$ for both). However, using the 2014 cefazolin breakpoints for urinary tract infections, $81.2 \%$ of the urine isolates were susceptible.

Susceptibilities of isolates from different specimen types were mostly similar but outpatient isolates were more susceptible than inpatient isolates. The overall prevalence of ESBL- and AmpC-producers was $8.2 \%$ and $4.7 \%$, respectively, but AmpC carriage increased significantly over the years (from 0 to $7.0 \%, P<0.001$ ). ESBL and AmpC $\beta$-lactamase-producers were more likely to be found in elderly and ICU patients. The predominant ESBL and AmpC $\beta$-lactamase genes were CTX-M- and CMY- types, respectively.

Conclusions: A significant decrease in susceptibility to 3rd-generation cephalosporins and ciprofloxacin occurred in P. mirabilis from Taiwan in the past decade. The prevalence of ESBL remained stable but AmpC $\beta$-lactamase-producing P. mirabilis increased significantly. Cefotaxime was a better surrogate than ceftazidime for predicting the presence of these $\beta$-lactamases. Continuous surveillance on antimicrobial resistance and associated resistance mechanisms in P. mirabilis is warranted.
\end{abstract}

Keywords: Drug resistance, Extended-spectrum $\beta$-lactamase, AmpC $\beta$-lactamase, Proteus mirabilis, Clinical and Laboratory Standards Institute (CLSI)

\footnotetext{
* Correspondence: lauderdale@nhri.org.tw

${ }^{2}$ National Institute of Infectious Diseases and Vaccinology, National Health Research Institutes, No. 35 Keyan Road, Zhunan 35053, Taiwan

Full list of author information is available at the end of the article
} 


\section{Background}

Proteus mirabilis belongs to the Enterobacteriaceae family with the features of swarming motility and production of urease to generate ammonia $[1,2]$. It can be found in soil, water, and the intestinal tract of mammals, including humans. In addition to being a leading cause of urinary tract infections (UTI), P. mirabilis can cause respiratory and wound infections, bacteremia, and other infections $[1,2]$. Although P. mirabilis is usually not a common cause of UTI among immunocompetent individuals, it is an important pathogen among patients with complicated urinary tract, urolithiasis, or long-term urinary catheterization [3]. Patients with UTI caused by P. mirabilis usually have alkaline $\mathrm{pH}$ urine due to the presence of ammonia resulting in calcium and magnesium crystallization which could in turn lead to obstruction of the lumen of indwelling catheters [4].

P. mirabilis was susceptible to cephalosporins and $\beta$-lactam $/ \beta$-lactamase inhibitors. However, strains resistant to $\beta$-lactams mediated by acquired $\beta$-lactamases emerged in 1990s [5]. Among these $\beta$-lactamases, plasmid-borne extended-spectrum $\beta$-lactamases (ESBL) and AmpC $\beta$-lactamases are most worrisome because they result in resistance to nearly all penicillins and cephalosporins and can spread among different species of Enterobacteriaceae [6]. Several other studies have shown that ESBL and AmpC $\beta$-lactamase-producing $P$. mirabilis isolates could lead to clonal spread and then cause intra-hospital, regional, and continent-wide outbreaks $[7,8]$. Treatment failure and clinical mortality are also more likely to occur in patients infected with ESBLs-producing P. mirabilis [9], which has been attributed to inadequate empirical therapy. The emergence and global spread of carbapenemaseproducing Enterobacteriaceae (CPE) in recent years, especially isolates carrying genes encoding KPC (Klebsiella pneumoniae carbapenemase) and NDM (New Delhi metallo- $\beta$-lactamase) carbapenemases, have further compromised treatment options and added to the crisis of antimicrobial resistance $[10,11]$. However, recent studies from different regions have found the prevalence of carbapenemase-producing P. mirabilis remained low [12-14].

Studies from the United States, Canada, United Kingdom, and other European countries revealed that susceptibility of $P$. mirabilis isolated from different sources can vary widely. For example, susceptibility to $\beta$-lactam/ $\beta$-lactamase inhibitors (ampicillin/sulbactam or amoxicillin/clavulanate), ciprofloxacin, and third generation cephalosporins (cefotaxime, ceftriaxone, or ceftazidime) ranged 74 to $94 \%, 60$ to $90 \%$, and 90 to $99 \%$, respectively, depending on patient population and specimen type $[12,15-18]$. Therefore, surveillance on the in vitro susceptibilities of $P$. mirabilis in each region is clinically relevant and important.
In addition, the Clinical and Laboratory Standards Institute (CLSI) revised the interpretive criteria on several $\beta$-lactams for Enterobacteriaceae in recent years. In 2010, CLSI lowered the aztreonam and 3rd-generation cephalosporin breakpoints for Enterobacteriaceae to facilitate the identification of isolates expressing ESBLs and/or AmpC $\beta$-lactamases. Breakpoints for different carbapenems were updated in June 2010 and in 2012. The most recent revision for cefazolin breakpoints occurred in 2011 and in 2014 (for isolates from urine only). Cefepime breakpoints were also revised in 2014. These changes are summarized in the 2014 CLSI M100-S24 document [19]. Of noteworthy also is that Proteus spp. can have naturally higher imipenem MICs $[19,20]$. Therefore, the new CLSI carbapenem breakpoints can have significant effect on the rate of imipenem susceptibility in $P$. mirabilis. To date, the impact of these changes on the susceptibilities to 3rd-generation cephalosporins and the correlation with carriage of ESBL and/or AmpC $\beta$-lactamases, and the effect on cefazolin and carbapenem susceptibility in $P$. mirabilis have seldom been discussed [21].

In Taiwan, $P$. mirabilis remains an important pathogen causing UTI among patients with urolithiasis and urinary catheters in both community and healthcare settings [22]. However, national data on the susceptibilities of $P$. mirabilis from different sources are limited. The Taiwan Surveillance of Antimicrobial Resistance (TSAR) is a biennial nationwide program conducted at the National Health Research Institutes (NHRI) [23]. The present study analyzed the TSAR data from period III (2002) to VIII (2012) to address the above issues.

\section{Methods}

\section{Isolate collection}

P. mirabilis isolates were collected as part of the TSAR program. Isolates were collected biennially between 2002 and 2012 (corresponding to TSAR III - VIII). For TSAR III to VII, isolates were collected from the same 26 hospitals (11 medical centers and 15 regional hospitals) except in 2008, when one hospital did not participate. In TSAR VIII (2012), isolates were collected from 25 of these 26 hospitals and two additional hospitals. These hospitals are located in the four geographic regions of Taiwan and all are general hospitals (Figure 1). The isolates were collected between July and September during the collection year and the collection process has been described previously [23]. Isolates were collected sequentially without specifying species to be collected. All isolates were stored at $-70^{\circ} \mathrm{C}$ in bead-containing Microbank cryovials (PRO-LAB Diagnostics, Austin, TX, USA) (for 2002 to 2006) or glycerol (20\%) containing trypticase soy broth (for 2008 to 2012). The bacterial isolates were recovered from clinical samples taken as part of standard care. The study was approved by the Research 


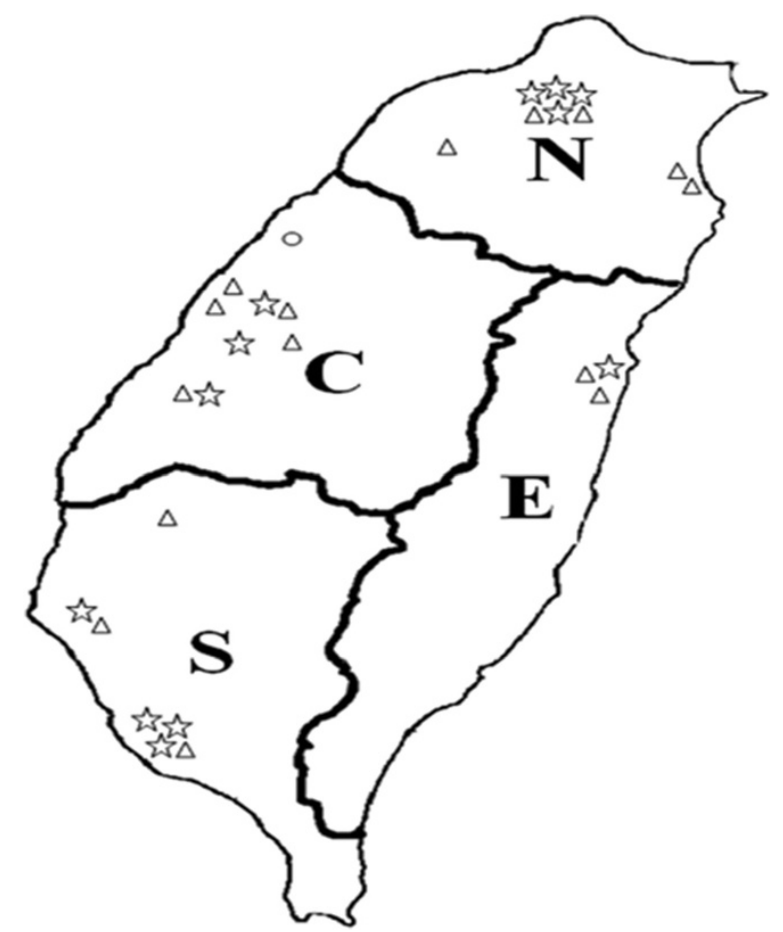

Figure 1 Distribution of hospitals that participated in the Taiwan Surveillance of Antimicrobial Resistance (TSAR) program from 2002 (TSAR III) to 2012 (TSAR VIII). The proximate locations of the hospitals are shown in each region ( $N$, north; $C$, central; $S$, South; E, East). Taiwan is a mountainous island and the majority of the people live in the most densely populated western part (north, central and south regions) while the eastern part is the least populated region. Hospital type: star, medical center; triangle, regional hospital; circle, local hospital, which participated in TSAR VIII (2012) only.

Ethics Committee of National Health Research Institutes (NHRI) (EC960205 and EC1010602-E).

\section{Isolate identification}

Isolates reported as $P$. mirabilis by hospitals were subcultured to blood agar and MacConkey agar plates for purity check and to confirm species identification at NHRI. Species identification was based on colony morphology and conventional biochemical reactions. For isolates with colony morphology or any biochemical reactions not typical of $P$. mirabilis, either Vitek I (prior to 2008) or Vitek II (2008 to 2012) GN cards were used (bioMérieux, Marcy l'Etoile, France).

\section{Antimicrobial susceptibility testing (AST)}

Minimum inhibitory concentrations (MICs) were determined by the broth microdilution method following the guidelines of the manufacturer and Clinical and Laboratory Standards Institute (CLSI) [24]. Sensititre custom-designed plates were used from 2002 to 2008, and the standard GNX2F plates were used in 2010 and 2012 [ThermoFisher Scientific (formerly Trek Diagnostics), East Grinstead, UK].
All isolates were subcultured twice on sheep blood agar plates from $-70^{\circ} \mathrm{C}$ prior to AST. Quality control was performed on each day of test with Escherichia coli ATCC 25922, E. coli ATCC 35218, and Pseudomonas aeruginosa ATCC 27853. The agents below were tested on isolates from all years: cefotaxime, ceftazidime, cefepime, aztreonam, imipenem, amikacin, gentamicin, ciprofloxacin, and trimethoprim/sulfamethoxazole (SXT). The following agents were tested on isolates from 2002 to 2008: ampicillin, amoxicillin/clavulanate, piperacillin, cefazolin, cefuroxime, cefoxitin; nitrofurantoin. In addition, ertapenem and meropenem were tested on isolates from 2010 and 2012. Interpretive criteria are based on the 2014 CLSI breakpoints. The impact of CLSI breakpoint revisions on susceptibilities to aztreonam, cefazolin, cefepime, cefotaxime, ceftazidime, ertapenem, imipenem, and meropenem are also compared using the current breakpoints and those of 2009, the year prior to different revisions [25].

\section{Determination of carriage of ESBL and/or AmpC $\beta$-lactamases genes}

All isolates with aztreonam, ceftazidime, or cefotaxime $\mathrm{MIC} \geq 2 \mathrm{mg} / \mathrm{L}$ were tested for the presence of ESBL by CLSI confirmatory test using cefotaxime and ceftazidime with and without clavulanic acid [25]. These isolates were also subjected to ESBL and/or AmpC $\beta$-lactamases gene detection by PCR. For each tested isolate, DNA extraction was performed using the following procedures. Three to five colonies were lightly picked from fresh overnight culture plate to suspend in $150 \mu \mathrm{l} \mathrm{AE}$ buffer. The suspension was heated at $95^{\circ} \mathrm{C}$ for $15 \mathrm{~min}$ then centrifuged at $1000 \mathrm{~g}$ for $10 \mathrm{~min}$ to remove cellular debris, after which $100 \mu \mathrm{l}$ of the supernatant was transferred to a new vial. The DNA preparation was stored at $-20^{\circ} \mathrm{C}$ and used as template for subsequent amplifications. Multiplex PCR was used to detect the relevant genes following previously described primers and protocols [26,27].

\section{Data analysis}

Susceptibility interpretation analysis was made using the WHONET software [28]. Duplicate isolates were excluded from analysis. The chi-square test was used for trend analysis on susceptibility to different agents over the years. Significance of differences in rates of susceptibility was tested by the $\chi 2$ test or Fisher's exact test (if the number was less than 10). The variable tested included $\beta$-lactam agents having old and revised CLSI breakpoints, and on isolates from different specimen types (blood, urine, pus/abscess, sputum) and patient locations [inpatients: intensive care units (ICU) or non-ICU, and outpatients]. Multivariable logistic regression analysis was performed to assess the variables (including study year, specimen type, patient age group, and patient location) among ESBL or AmpC $\beta$-lactamase -producers vs. -non-producers. 
All analyses were performed using SAS 9.2 (SAS Institute, Cary, NC, USA). A 2-tailed $P$ value less than 0.05 was considered statistically significant.

\section{Results}

Isolates

A total of $1,157 P$. mirabilis isolates were collected by the TSAR program with 176, 186, 205, 219, 185, and 186 isolates from 2002, 2004, 2006, 2008, 2010, and 2012, respectively. The most common specimen type was urine, accounting for $49.4 \%$ (571), followed by pus/abscess (233, 20.1\%), blood (158, 13.7\%), sputum (133, 11.5\%), and others $(62,5.4 \%)$. The age of the source patients was known in 1110 patients. The mean age of the patients was $62.8 \pm 24.7$ years, with $9.2 \%$ being pediatric patients ( $\leq 18$ years), $29.6 \%$ being adult (19-64 years), and 61.3\% being elderly ( $\geq 65$ years). Most isolates were from inpatients (65.2\%; 53.6\% in general ward, and $11.6 \%$ in ICU).

\section{Susceptibility to different antimicrobial agents over the} years and the impact of different CLSI breakpoints

The susceptibilities of the 1157 isolates to various antibiotics by year are presented in Table 1. Significant decrease in susceptibilities over the years (from 2002 to 2012) to cefotaxime (from $92.6 \%$ to $81.7 \%$ ), ceftazidime (from 100\% to $95.2 \%$ ), and ciprofloxacin (from $80.1 \%$ to $53.8 \%$ ) occurred.

Table 1 Antimicrobial susceptibilities (\%) of Proteus mirabilis by study year, 2002-2012

\begin{tabular}{|c|c|c|c|c|c|c|c|c|}
\hline Antimicrobial agents $^{a}$ & $\begin{array}{c}2002 \\
(n=176)\end{array}$ & $\begin{array}{c}2004 \\
(n=186)\end{array}$ & $\begin{array}{c}2006 \\
(n=205)\end{array}$ & $\begin{array}{c}2008 \\
(n=219)\end{array}$ & $\begin{array}{c}2010 \\
(n=185)\end{array}$ & $\begin{array}{c}2012 \\
(n=186)\end{array}$ & $P^{\mathbf{b}}$ & $\begin{array}{c}\text { total } \\
(n=1157)\end{array}$ \\
\hline \multicolumn{9}{|l|}{$\beta$-lactams: } \\
\hline Amoxicillin/CA ${ }^{c}$ & 91.4 & 74.7 & 86.8 & 83.6 & NT & NT & & 84.6 \\
\hline Ampicillin & 33.3 & 32.3 & 31.7 & 39.3 & NT & NT & & 34.3 \\
\hline Aztreonam_2009 & 99.4 & 99.5 & 98.5 & 100 & 100 & 99.5 & & 99.5 \\
\hline Aztreonam & 99.4 & 98.9 & 98.5 & 100 & 100 & 98.9 & & 99.3 \\
\hline Cefazolin_2009 & 73.6 & 64.0 & 74.2 & 75.3 & NT & NT & & $71.9^{\mathrm{d}}$ \\
\hline Cefazolin & 0.6 & 0 & 2.4 & 0.5 & NT & NT & & 0.9 \\
\hline Cefuroxime & 92.5 & 83.3 & 85.9 & 88.6 & NT & NT & & 87.5 \\
\hline Cefotaxime_2009 & 95.5 & 88.2 & 96.6 & 94.9 & 98.4 & 97.3 & & 95.2 \\
\hline Cefotaxime & 92.6 & 84.4 & 86.8 & 88.5 & 80 & 81.7 & 0.003 & 85.7 \\
\hline Ceftazidime_2009 & 100 & 99.5 & 99 & 98.2 & 97.8 & 95.7 & 0.001 & 98.4 \\
\hline Ceftazidime & 100 & 98.4 & 98.1 & 96.4 & 95.6 & 95.2 & 0.001 & 97.2 \\
\hline Cefoxitin & 96 & 94.1 & 94.6 & 95 & NT & NT & & 94.9 \\
\hline Cefepime & 97.2 & 91.4 & 97.6 & 99.1 & 100 & 98.9 & & $97.4^{\mathrm{e}}$ \\
\hline Ertapenem_2009 & NT & NT & NT & NT & 100 & 100 & & 100 \\
\hline Ertapenem & NT & NT & NT & NT & 100 & 99.5 & & 99.7 \\
\hline Imipenem_2009 & 100 & 100 & 99.5 & 99.5 & 100 & 100 & & $99.8^{d}$ \\
\hline Imipenem & 34.7 & 16.1 & 56.6 & 43.4 & 92.4 & 67.2 & & 51.7 \\
\hline Meropenem & NT & NT & NT & NT & 100 & 100 & & 100 \\
\hline Piperacillin & 48.9 & 44.6 & 37.6 & 43.3 & NT & NT & & 43.4 \\
\hline \multicolumn{9}{|l|}{ Non $\beta$-lactams: } \\
\hline Amikacin & 92.6 & 88.2 & 89.8 & 90.4 & 90.3 & 88.7 & & 90 \\
\hline Gentamicin & 59.1 & 60.8 & 54.2 & 62.1 & 55.1 & 54.3 & & 57.7 \\
\hline Ciprofloxacin & 80.1 & 70.3 & 68.3 & 69.9 & 70.3 & 53.8 & $<0.001$ & 68.7 \\
\hline $\mathrm{TMP} / \mathrm{SMX}(\mathrm{SXT})^{\mathrm{C}}$ & 35.8 & 33.3 & 29.8 & 37 & 36.8 & 31.7 & & 34.1 \\
\hline ESBL prevalence & 5.1 & 10.2 & 10.7 & 6.9 & 5.4 & 10.8 & & 8.2 \\
\hline AmpC prevalence & 0 & 3.8 & 2.9 & 5.0 & 9.2 & 7.0 & $<0.001$ & 4.7 \\
\hline
\end{tabular}

${ }^{a}$ Susceptibility results are based on the current CLSI breakpoints [19]. For agents with breakpoint revision in recent years, results of the 2009 CLSI criteria are also shown for comparison [25].

${ }^{\mathrm{b}}$ Chi-square for trends. Only statistically significant results are shown.

${ }^{c}$ Amox/CA, Amoxacillin/clavulanic acid; TMP/SMX (SXT), trimethoprim/sulfamethoxazole.

${ }^{d}$ Italicized, significant difference $(P<0.001)$ in susceptibility rates using old and revised breakpoints.

${ }^{\text {e}}$ Cefepime results include $3.5 \%$ (41 isolates) in the SDD (susceptible dose dependent) category. 
The revised CLSI breakpoints impacted significantly (overall susceptibility 2009 vs. current, $P<0.001$ ) on susceptibilities to cefazolin $(71.9 \%$ vs. $0.9 \%)$, cefotaxime ( $95.2 \%$ vs. $85.7 \%)$, and imipenem (99.8\% vs. 55.1\%). The MIC distribution of cefazolin and imipenem are shown in Figure 2 to illustrate how the breakpoint change affected the susceptibility rate. Using the 2009 vs. current breakpoints, 9\% vs. 52.2\% and $19.1 \%$ vs. $46.9 \%$ of the isolates had cefazolin MIC in the intermediate $(4 \mathrm{mg} / \mathrm{L})$ and resistant $(>=8 \mathrm{mg} / \mathrm{L})$ range, respectively. For imipenem, the revised MIC breakpoints resulted in $35.6 \%$ and $12.7 \%$ of the isolates in the intermediate $(2 \mathrm{mg} / \mathrm{L})$ and resistant (> $=4 \mathrm{mg} / \mathrm{L})$ category, respectively. The revised breakpoints did not have significant effect on the susceptibility to ceftazidime ( 98.4 vs. $97.2 \%)$, aztreonam (99.5 vs. 99.3\%), ertapenem (100 vs. 99.7\%), and meropenem (all 100\%) (Table 1).

\section{Susceptibility of isolates from different sources}

Some variations in rates of susceptibility to individual agents existed for isolates from different specimen types and patient locations (Table 2). By specimen types, isolates recovered from sputum had significantly lower susceptibility to cefazolin (by CLSI 2009 criteria), piperacillin, and trimethoprim/sulfamethoxazole compared to other specimen types. For urine isolates, susceptibility to cefazolin differed significantly using the CLSI 2009 and 2014 updated UTI criteria ( 81.2 vs. $74.3 \%, P=0.016)$ (Table 2$)$. Isolates from outpatients had significantly higher rates of susceptibility to ampicillin, amoxicillin/clavulanate, cefazolin (by CLSI 2009 criteria), cefuroxime, cefotaxime, ciprofloxacin, gentamicin, and piperacillin.

\section{Prevalence of ESBL and AmpC $\beta$-lactamases genes}

There were 172 isolates with ESBL phenotype (aztreonam, ceftazidime, and/or cefotaxime MICs $\geq 2 \mathrm{mg} / \mathrm{L}$ ). Among them, 95 carried ESBL genes and 54 carried AmpC genes including 7 that carried both ESBL and AmpC genes. For the isolates carrying ESBL genes, 92 carried CTX-M-type genes, two carried SHV-type genes, and one carried both SHV and CTX-M genes. For the isolates carrying AmpC genes, 48 carried CMY-type genes, and 6 carried DHA-type genes. By Chi-square for trends, the prevalence of ESBL did not increase significantly during the study period (5.1 - 10.8\%, $P=0.607$; Table 1). However, the prevalence of AmpC $\beta$-lactamases gene carriage increased significantly (from 0 to $7.0 \%, P<0.001$; Table 1 ).

\section{Susceptibilities of isolates carrying ESBL and AmpC $\beta$-lactamases genes}

Using the current CLSI breakpoints, none of 142 isolates with either ESBL or AmpC gene was susceptible to cefotaxime [sensitivity, 100\%; specificity, 97.5\%; positive predictive value (PPV), 86.1\%; negative predictive value (NPV), 100\%]. However, $79.6 \%$ of the 142 isolates were susceptible to ceftazidime (sensitivity, 20.4\%; specificity, 99.7\%; PPV, 68.8\%; NPV, 90.0\%) and $95.8 \%$ were susceptible to aztreonam (sensitivity, 4.2\%; specificity, 99.5\%; PPV, 75\%; NPV, 88.2\%). Compared to non-ESBL phenotype and ESBL- and AmpC- negative isolates, ESBL and AmpC $\beta$-lactamase-producing isolates had significantly lower rates of susceptibility to amikacin $(28.4 \%-57.1 \%$ vs. $97.8 \%)$, cefotaxime (0 vs. 99.7\%), and ciprofloxacin (6.8\% - 42.9\% vs. $75.9 \%$ ) (Table 3). Of note, near $40 \%$ of the ESBL positive

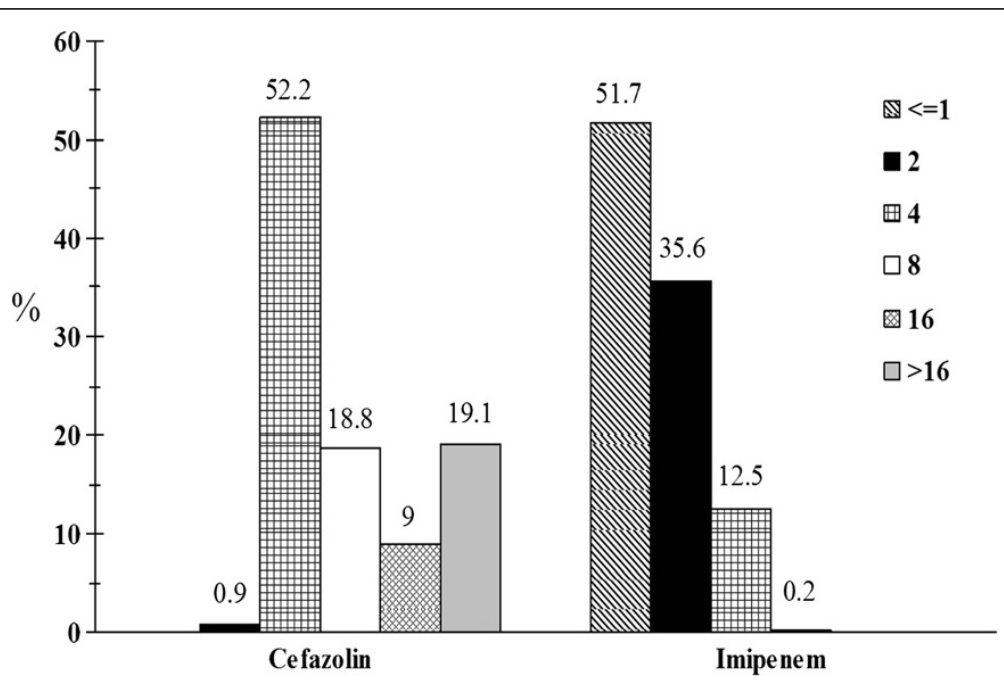

Figure 2 MIC distributions of cefazolin and imipenem in Proteus mirabilis. Data for cefazolin and imipenem are from 786 and 1157 isolates, respectively (cefazolin was not tested in 2010 and 2012) in the Taiwan Surveillance of Antimicrobial Resistance (TSAR) 2002 to 2012 program. The susceptible, intermediate, and resistant breakpoints (2009 vs. current) are $<=8,16$, and $>=32 \mathrm{vs}$. $\langle=2,4$, and $>=8 \mathrm{mg} / \mathrm{L}$ for cefazolin; and $<=4,8$, and $>=16$ vs. $<=1,2$, and $>=4 \mathrm{mg} / \mathrm{L}$ for imipenem. Numbers on top of the bars represent $\%$ of isolates with that MIC value. 
Table 2 Antimicrobial susceptibilities of Proteus mirabilis by specimen types and patient locations

\begin{tabular}{|c|c|c|c|c|c|c|c|}
\hline \multirow[t]{2}{*}{ Antimicrobial agents ${ }^{\mathrm{a}}$} & \multicolumn{4}{|c|}{ Specimen types ${ }^{b}$} & \multicolumn{3}{|c|}{ Patient locations } \\
\hline & $\begin{array}{c}\text { Urine } \\
(n=571)\end{array}$ & $\begin{array}{c}\text { Pus/abscess } \\
(n=233)\end{array}$ & $\begin{array}{c}\text { Blood } \\
(n=158)\end{array}$ & $\begin{array}{l}\text { Sputum } \\
(n=133)\end{array}$ & $\begin{array}{c}\text { ICU } \\
(n=134)\end{array}$ & $\begin{array}{l}\text { Non-ICU } \\
(n=618)\end{array}$ & $\begin{array}{l}\text { Outpatients } \\
(n=401)\end{array}$ \\
\hline \multicolumn{8}{|l|}{ B-lactams: } \\
\hline Amoxicillin/CA & 83.2 & 87.1 & 88.9 & 76.9 & $72.3^{d}$ & 83.9 & 88.9 \\
\hline Ampicillin & 33.1 & 36.2 & 44.4 & 25.3 & 33.0 & 31.7 & 40.2 \\
\hline Cefazolin_2009 & 74.3 & 73.6 & 77.8 & 50.6 & 58.5 & 69.2 & 81.6 \\
\hline Cefazolin & 1.2 & 1.2 & 0 & 0 & 1.1 & 0.7 & 1.2 \\
\hline Cefazolin_UTI| & 81.2 & - & - & - & 64.7 & 79.5 & 86.5 \\
\hline Cefuroxime & 86.9 & 91.4 & 90 & 80.2 & 78.7 & 86.9 & 92.0 \\
\hline Cefotaxime & 85.6 & 89.2 & 84.8 & 80.3 & 80.6 & 84.6 & 89.3 \\
\hline Ceftazidime & 98.1 & 97.9 & 95.6 & 94.9 & 96.3 & 97.2 & 97.5 \\
\hline Cefoxitin & 94.1 & 96.9 & 94.4 & 94.5 & 91.5 & 95.1 & 96.2 \\
\hline Cefepime $^{f}$ & 97.0 & 98.3 & 99.4 & 95.6 & 95.5 & 97.1 & 98.5 \\
\hline Imipenem_2009 & 100 & 100 & 100 & 100 & 99.3 & 100 & 100 \\
\hline Imipenem & 34.7 & 16.1 & 56.6 & 43.8 & 53.0 & 50.2 & 53.8 \\
\hline Piperacillin & 41.4 & 51.2 & 47.8 & 29.7 & 41.5 & 38.2 & 52.7 \\
\hline \multicolumn{8}{|l|}{ Non $\beta$-lactams: } \\
\hline Amikacin & 90.5 & 93.6 & 87.3 & 84.7 & 85.1 & 89.8 & 92 \\
\hline Gentamicin & 59.5 & 63.5 & 58.9 & 35.8 & 52.2 & 53.9 & 65.8 \\
\hline Ciprofloxacin & 69.2 & 70.8 & 67.1 & 64.2 & 62.7 & 66.5 & 74.6 \\
\hline TMP/SMX $(S X T)^{c}$ & 33.1 & 40.3 & 34.8 & 24.8 & 35.1 & 31.9 & 37.4 \\
\hline
\end{tabular}

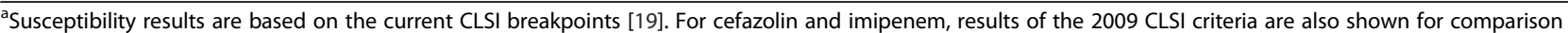
[25]. Other agents with overall susceptibility $>99 \%$ (aztreonam, ertapenem, meropenem) are not shown.

${ }^{b}$ Data on miscellaneous other specimen types $(n=63)$ are not shown.

${ }^{c}$ Amox/CA, Amoxacillin/clavulanic acid; TMP/SMX (SXT), trimethoprim/sulfamethoxazole.

d Italicized, significant difference in susceptibility rate compared to other subgroups.

${ }^{e}$ Result of urine isolates are shown using the 2014 UTI breakpoints [19].

fCefepime results include SDD (susceptible dose dependent) category.

Table 3 Susceptibilities to key agents among the Proteus mirabilis isolates with and without ESBL or AmpC

$\beta$-lactamases genes

\begin{tabular}{lcccc}
\hline Antimicrobial agent $^{\mathbf{a}}$ & \multicolumn{3}{c}{ Susceptibility (\%) } \\
\cline { 2 - 5 } & $\begin{array}{c}\text { ESBL(+)/AmpC(+) } \\
(\mathbf{n}=\mathbf{7})\end{array}$ & $\begin{array}{c}\text { ESBL(+)/AmpC(-) } \\
(\mathbf{n}=\mathbf{8 8})\end{array}$ & $\begin{array}{c}\text { ESBL(-)/AmpC(+) } \\
(\mathbf{n}=\mathbf{4 7})\end{array}$ & $\begin{array}{c}\text { Non-ESBL phenotype \& ESBL(-)/AmpC(-) } \\
(\mathbf{n}=\mathbf{1 0 1 5})\end{array}$ \\
\hline Amikacin & 57.1 & 28.4 & 40.4 & 97.8 \\
Aztreonam & 100 & 94.3 & 97.9 & 99.8 \\
Cefepime (S/SDD) & $42.85 / 42.85$ & $29.5 / 38.6$ & $91.5 / 6.4$ & $99.9 / 0.1$ \\
Cefotaxime & 0 & 0 & 0 & 99.7 \\
Ceftazidime & 71.4 & 94.3 & 53.2 & 99.7 \\
Ciprofloxacin & 42.9 & 6.8 & 34.0 & 75.9 \\
Ertapenem & $100(\mathrm{n}=4)$ & $100(\mathrm{n}=26)$ & $100(\mathrm{n}=26)$ & $99.7(\mathrm{n}=315)$ \\
Imipenem_2009 & 100 & 100 & 100 & 99.9 \\
Imipenem & 42.9 & 45.5 & 66.0 & 51.7
\end{tabular}

${ }^{a}$ Susceptibility results are based on the current CLSI breakpoints [19]. For imipenem, results of the 2009 CLSI criteria are also shown for comparison [25]. All were susceptible to meropenem.

${ }^{\mathrm{b}}$ Cefepime results are shown in susceptible and SDD (susceptible dose dependent) categories. 
isolates had cefepime MIC in the susceptible dose dependent (SDD) range compared with $6.4 \%$ of the AmpC-positive only isolates (Table 3 ).

\section{Factors associated with ESBL and AmpC $\beta$-lactamases gene carriage}

Factors associated with carriage of ESBL included age and patient location using univariate analysis (Table 4). By multivariate analysis, age [elderly vs. adult patients; odds ratio (OR), 3.85; 95\% confidence interval (95\% C.I.), 2.02-7.33; $P<0.001$ ] and patient location (ICU vs. outpatients; OR, $1.99 ; 95 \%$ C.I., 1.04-3.84; $P=0.039$ ) remained independent factors associated ESBL genes carriage. Age (elderly vs. adult patients; OR, 2.22; 95\% C.I., $1.06-4.62 ; P=0.034$ ) was also a sole factor significantly associated with AmpC $\beta$-lactamase gene carriage by both univariate and multivariate analyses.

\section{Discussion}

This multicenter longitudinal surveillance revealed significant decreased susceptibility to cefotaxime, ceftazidime, and ciprofloxacin occurred in P. mirabilis from Taiwan in the past decade. In addition, compared to recent reports from the United States, Canada, and United Kingdom, our results showed that $P$. mirabilis in Taiwan have lower rates of susceptibility to cefotaxime ( $85.7 \%$ vs. > $97 \%$ in US, Canada, and UK) and gentamicin (57.7\% vs. > 90\%)
[12,15-17]. Susceptibility to ciprofloxacin was also much lower than rates found in the United States and Canada $(68.7 \%$ vs. > $80 \%)[16,17]$.

The lower rate of cefotaxime-susceptibility in our isolates might be due to a higher prevalence of ESBL- and/or AmpC $\beta$-lactamase- producers. Our overall 8.2\% ESBL rate is higher than the $<5 \%$ reported on $P$. mirabilis from different sources in the United States [15,16,29]. In contrast to ESBL-producers, data on changes in AmpC prevalence in P. mirabilis are scarce. The increase of AmpC $\beta$-lactamase-producers over the years observed in the present study may be due to clonal spread and horizontal gene transfer. The lower and decreased susceptibility to ciprofloxacin over the study periods might be due to increased consumption of fluoroquinolones in Taiwan in recent years [30].

Although the prevalence of ESBL-producers remained stable, AmpC $\beta$-lactamase-producers increased significantly over the study years. Whether this phenomenon was due to clonal spread or horizontal gene transfer requires further study. Most ESBL genes were CTX-M types (94.8\%). The predominant AmpC $\beta$-lactamases genes were CMY-types (88.9\%), with the rest being DHA-type. Studies from Taiwan on other species of Enterobacteriaceae have found CTX-M-type ESBL and CMY-type and DHA-type AmpC $\beta$-lactamases to be prevalent, including Escherichia coli, Klebsiella pneumoniae, and Enterobacter spp. [14]. Of

Table 4 Univariate analysis for factors associated with carriage of ESBL and AmpC $\beta$-lactamases genes in Proteus mirabilis

\begin{tabular}{|c|c|c|c|c|c|c|c|c|}
\hline \multirow[t]{3}{*}{ Variables } & \multicolumn{4}{|c|}{ ESBL } & \multicolumn{4}{|c|}{ AmpC $\beta$-lactamases } \\
\hline & \multirow[t]{2}{*}{ Odds ratio } & \multicolumn{2}{|c|}{ 95\% confidence interval } & \multirow[t]{2}{*}{$P$} & \multirow[t]{2}{*}{ Odds ratio } & \multicolumn{2}{|c|}{$95 \%$ confidence interval } & \multirow[t]{2}{*}{$P$} \\
\hline & & Lower & Upper & & & Lower & Upper & \\
\hline \multicolumn{9}{|c|}{ Study year (using TSAR VIII [2012] as baseline) ${ }^{a}$} \\
\hline TSAR III (2002) & 0.45 & 0.20 & 1.01 & 0.053 & - & - & - & - \\
\hline TSAR IV (2004) & 0.94 & 0.49 & 1.83 & 0.866 & 0.52 & 0.20 & 1.34 & 0.174 \\
\hline TSAR V (2006) & 0.99 & 0.53 & 1.89 & 0.995 & 0.40 & 0.15 & 1.08 & 0.070 \\
\hline TSAR VI (2008) & 0.61 & 0.30 & 1.23 & 0.167 & 0.70 & 0.31 & 1.61 & 0.406 \\
\hline TSAR VII (2010) & 0.47 & 0.22 & 1.04 & 0.064 & 1.35 & 0.63 & 2.86 & 0.438 \\
\hline \multicolumn{9}{|c|}{ Age groups (using adult as baseline) ${ }^{b}$} \\
\hline Pediatric patients & 0.29 & 0.04 & 2.24 & 0.233 & 1.07 & 0.29 & 4.05 & 0.916 \\
\hline Elderly patients & 4.01 & 2.11 & 7.63 & $<0.001$ & 2.22 & 1.06 & 4.62 & 0.034 \\
\hline \multicolumn{9}{|c|}{ Specimen types (using blood as baseline) } \\
\hline Urine & 0.90 & 0.48 & 1.69 & 0.745 & 0.89 & 0.40 & 2.02 & 0.788 \\
\hline Sputum & 0.56 & 0.25 & 1.24 & 0.153 & 0.75 & 0.28 & 2.00 & 0.569 \\
\hline Pus/abscess & 1.56 & 0.74 & 3.26 & 0.241 & 1.48 & 0.57 & 3.85 & 0.426 \\
\hline others & 0.97 & 0.33 & 2.83 & 0.956 & 0.33 & 0.04 & 2.69 & 0.300 \\
\hline \multicolumn{9}{|c|}{ Patients' location (using outpatient as baseline) } \\
\hline Non-ICU & 2.46 & 1.29 & 4.70 & 0.006 & 1.07 & 0.41 & 2.77 & 0.890 \\
\hline ICU & 2.23 & 0.99 & 4.98 & 0.051 & 1.21 & 0.66 & 2.21 & 0.545 \\
\hline
\end{tabular}

${ }^{a}$ There were no AmpC $\beta$-lactamase gene positive isolates in TSAR III (2002).

${ }^{b}$ Definition of age groups for pediatric, adult, and elderly patients was $<=18,19-64$, and $>=65$ year olds, respectively. 
note, there was only one isolate with non-susceptibility to ertapenem $(\mathrm{MIC}=1 \mathrm{mg} / \mathrm{L})$. Therefore, carbapenemases do not currently appear to be prevalent in P. mirabilis in Taiwan.

Our results also showed that the revised CLSI breakpoints have significant impact on susceptibility to cefazolin (2009 vs. current breakpoints, $71.9 \%$ vs. $0.9 \%$ ), cefotaxime (95.2\% vs. $85.7 \%)$, and imipenem (99.8\% vs. $51.8 \%)$ in $P$. mirabilis $(P<0.001)$. The marked decrease in susceptibility to cefazolin is due in part to a large number (52.2\%) of isolates falling into the immediate category (MIC $4 \mathrm{mg} / \mathrm{L}$ ). A study from Canada also showed similar MIC distribution in P. mirabilis, with $4.2 \%$ and $63.6 \%$ of isolates in the susceptible and intermediate category, respectively [17]. However, using the 2014 UTI interpretive criteria for cefazolin, $81.2 \%$ of the urine isolates in our study were susceptible. This information can be helpful in therapy selection for uncomplicated UTIs.

The large difference in imipenem susceptibility when using different breakpoints (2009 vs. current, $99.8 \%$ vs. $51.8 \%$ ) is because of the high percentages of $P$. mirabilis isolates having imipenem MICs of either 2 or $4 \mathrm{mg} / \mathrm{L}$, which were considered susceptible by the old criteria but intermediate and resistant, respectively, by the new criteria. It is well known that $P$. mirabilis can present with higher MICs against imipenem compared to other carbapenem agents [19]. In fact, among the 373 P. mirabilis isolates that had ertapenem, imipenem, and meropenem tested in our study, 76 had imipenem MIC of either 2 or $4 \mathrm{mg} / \mathrm{L}$. These 76 isolates all had meropenem $\mathrm{MIC}<=1 \mathrm{mg} / \mathrm{L}, 75$ of which had ertapenem MIC $<=0.25 \mathrm{mg} / \mathrm{L}$ and only one isolate had ertapenem MIC $1 \mathrm{mg} / \mathrm{L}$ (data not shown). Therefore, applying the revised CLSI criteria would result in significantly fewer carbapenem-susceptible $P$. mirabilis being reported. Similar results have been found on P. mirabilis isolated from ICUs in Taiwan [21]. In the European Committee on Antimicrobial Susceptibility Testing MIC distribution database, $63.8 \%$ of the tested P. mirabilis $(\mathrm{n}=15852)$ had an imipenem $\mathrm{MIC}<=1 \mathrm{mg} / \mathrm{L}$, yet $97.8 \% \mathrm{had}<=4 \mathrm{mg} / \mathrm{L}$ [31]. In two recent studies using the revised breakpoints, low rates of susceptibility to imipenem in P. mirabilis (9\% and $26.5 \%$ ) were noted $[15,16]$. It has been reported that susceptibility of $P$. mirabilis to carbapenems should be determined by ertapenem, meropenem, or doripenem [21,32]. Our study echoed this suggestion.

CLSI lowered the susceptibility breakpoints of Enterobacteriaceae, including P. mirabilis, for 3rdgeneration cephalosporins in 2010 to facilitate the identification of isolates having ESBLs and/or AmpC $\beta$-lactamases [33]. Using the revised breakpoints, none of the ESBL and/or AmpC positive isolates was susceptible to cefotaxime, yet $53.2 \%$ to $94.3 \%$ remained susceptible to ceftazidime. Therefore cefotaxime is a better predictor for
ESBLs and/or AmpC $\beta$-lactamase-producers than ceftazidime in terms of sensitivity $(100 \%$ vs. $20.4 \%)$ and PPV (86.1\% vs. $68.8 \%)$. This echoed the findings of prior studies on other species of Enterobacteriaceae [34,35]. The higher sensitivity and PPV of cefotaxime reflects the presence of CTX-M since it hydrolyzes cefotaxime more efficiently than ceftazidime [36]. Of interest also is that using the 2014 revised cefepime breakpoints, around $40 \%$ of the ESBL-producers had cefepime MIC in the susceptible dose dependent range, indicating that higher dosing regimens are needed if cefepime was used for these isolates [19].

Independent factors associated with the presence of ESBL genes were age (elderly patients) and location (ICU) of source patients. The only independent factor associated with the presence of AmpC $\beta$-lactamase genes was age (elderly patients) of source patients. Both of these two factors implied higher prior antibiotic use and/or more broad-spectrum antibiotic exposure, which in turn can result in acquisition of drug-resistance genes.

One limitation of the present study is that isolates were collected biennially during a three months period. However, our isolates were from 28 hospitals located in all four regions of Taiwan, 25 of which participated in all 6 rounds of TSAR between 2002 and 2012. These 28 hospitals included 12 medical centers, 15 regional hospitals, and one local hospital. Therefore, the results presented here are a representation of the total number of $P$. mirabilis in Taiwan.

\section{Conclusion}

This multicenter surveillance revealed decreased susceptibility of $P$. mirabilis in Taiwan to some broad spectrum antibiotics, including 3rd-generation cephalosporins and ciprofloxacin, in the past decade. The prevalence of AmpC $\beta$-lactamase-producing $P$. mirabilis also increased significantly. Patient age and location were factors independently associated with the presence of ESBL and/or AmpC $\beta$-lactamase-producers. Cefotaxime was a better surrogate than ceftazidime for predicting the presence of ESBL and/or AmpC $\beta$-lactamases. Continuous surveillance on antimicrobial resistance and associated resistance mechanisms in P. mirabilis is warranted.

\section{Competing interests}

The authors declare that they have no competing interests.

\section{Authors' contributions}

JTW performed data analysis and interpretation, and drafted the manuscript. PCC, YRS, HYW, JFL, IWH, and MCC carried out the laboratory assays and participated in data analysis. SCC participated in the design and data interpretation of the study. TLL designed and supervised the study, participated in data analysis and interpretation, and finalized the manuscript. All authors read and approved the final version of the manuscript.

\section{Acknowledgements}

We thank the following 28 hospitals for participating in the Taiwan Surveillance of Antimicrobial Resistance (TSAR) program: Buddhist Tzu Chi General Hospital, Cathay General Hospital, Changhua Christian Hospital, 
Cheng-Ching Hospital, Chung Shan Medical University Hospital, Ditmanson Medical Foundation Chia-Yi Christian Hospital, Da Chien Health Medical System (TSAR VIII only), Far Eastern Memorial Hospital, Hua-Lien Hospital, Jen-Ai Hospital, Kaohsiung Armed Forces General Hospital, Kaohsiung Chang Gung Memorial Hospital of the C. G. M. F, Kaohsiung Medical University Chung-Ho Memorial Hospital, Kaohsiung Veterans General Hospital, Kuang Tien General Hospital, Lo-Hsu Foundation Inc., Lotung Poh-Ai Hospital, Mennonite Christian Hospital, Min-Sheng Healthcare, National Cheng Kung University Hospital, Saint Mary's Hospital Luodong, Show Chwan Memorial Hospital, Tungs' Taichung MetroHarbor Hospital, Taichung Veterans General Hospital, Tainan Sin-Lau Hospital - the Presbyterian Church in Taiwan, Taipei City Hospital Heping Fuyou Branch, Taipei City Hospital Zhongxiao Branch (not TSAR VIII), Taipei Veterans General Hospital (TSAR VIII only), Tri-Service General Hospital (not TSAR V). This project was supported by an intramural grant from the National Health Research Institutes (IVPP01-014).

\section{Author details}

'Division of Infectious Diseases, Department of Internal Medicine, National Taiwan University Hospital, 7 Chung-Shan S. Road, Taipei 10002, Taiwan. ${ }^{2}$ National Institute of Infectious Diseases and Vaccinology, National Health Research Institutes, No. 35 Keyan Road, Zhunan 35053, Taiwan. ${ }^{3}$ College of Medicine, National Taiwan University, 1 Jen-Ai Road Section 1, Taipei 10051, Taiwan.

Received: 13 April 2014 Accepted: 2 September 2014

Published: 5 September 2014

\section{References}

1. Mobley HL, Belas R: Swarming and pathogenicity of Proteus mirabilis in the urinary tract. Trends Microbiol 1995, 3:280-284.

2. Rozalski A, Sidorczyk Z, Kotelko K: Potential virulence factors of Proteus bacilli. Microbiol Mol Biol Rev 1997, 61:65-89.

3. Warren JW, Tenney JH, Hoopes JM, Muncie HL, Anthony WC: A prospective microbiologic study of bacteriuria in patients with chronic indwelling urethral catheters. J Infect Dis 1982, 146:719-723.

4. Kunin CM: Blockage of urinary catheters: role of microorganisms and constituents of the urine on formation of encrustations. J Clin Epidemiol 1989, 42:835-842.

5. Chanal C, Bonnet R, De Champs C, Sirot D, Labia R, Sirot J: Prevalence of $\beta$-lactamases among 1,072 clinical strains of Proteus mirabilis: a 2-year survey in a French hospital. Antimicrob Agents Chemother 2000, 44:1930-1935.

6. Bush K, Jacoby GA: Updated functional classification of $\beta$-lactamases. Antimicrob Agents Chemother 2010, 54:969-976.

7. D'Andrea MM, Literacka E, Zioga A, Giani T, Baraniak A, Fiett J, Sadowy E, Tassios PT, Rossolini GM, Gniadkowski M, Miriagou V: Evolution and spread of a multidrug-resistant Proteus mirabilis clone with chromosomal AmpC-type cephalosporinases in Europe. Antimicrob Agents Chemother 2011, 55:2735-2742.

8. Nakano R, Nakano A, Abe M, Inoue M, Okamoto R: Regional outbreak of CTX-M-2 $\beta$-lactamase-producing Proteus mirabilis in Japan. J Med Microbiol 2012, 61:1727-1735.

9. Endimiani A, Luzzaro F, Brigante G, Perilli M, Lombardi G, Amicosante G, Rossolini GM, Toniolo A: Proteus mirabilis bloodstream infections: risk factors and treatment outcome related to the expression of extended-spectrum $\beta$-lactamases. Antimicrob Agents Chemother 2005, 49:2598-2605.

10. Bush K: Alarming $\beta$-lactamase-mediated resistance in multidrug-resistant Enterobacteriaceae. Curr Opin Microbiol 2010, 13:558-564.

11. Nordmann P, Naas T, Poirel L: Global spread of carbapenemase-producing Enterobacteriaceae. Emerg Infect Dis 2011, 17:1791-1798.

12. Horner CS, Abberley N, Denton M, Wilcox MH: Surveillance of antibiotic susceptibility of Enterobacteriaceae isolated from urine samples collected from community patients in a large metropolitan area, 2010-2012. Epidemiol Infect 2014, 142:399-403.

13. Miro E, Aguero J, Larrosa MN, Fernandez A, Conejo MC, Bou G, GonzalezLopez JJ, Lara N, Martinez-Martinez L, Oliver A, Aracil B, Oteo J, Pascual A, Rodriguez-Bano J, Zamorano L, Navarro F: Prevalence and molecular epidemiology of acquired AmpC $\beta$-lactamases and carbapenemases in Enterobacteriaceae isolates from 35 hospitals in Spain. Eur J Clin Microbiol Infect Dis 2013, 32:253-259.
14. Sheng WH, Badal RE, Hsueh PR: Distribution of extended-spectrum $\beta$-lactamases, AmpC $\beta$-lactamases, and carbapenemases among Enterobacteriaceae isolates causing intra-abdominal infections in the AsiaPacific region: results of the Study for Monitoring Antimicrobial Resistance Trends (SMART). Antimicrob Agents Chemother 2013, 57:2981-2988.

15. Bouchillon SK, Badal RE, Hoban DJ, Hawser SP: Antimicrobial susceptibility of inpatient urinary tract isolates of gram-negative bacilli in the United States: results from the Study for Monitoring Antimicrobial Resistance Trends (SMART) program: 2009-2011. Clin Ther 2013, 35:872-877.

16. Hawser SP, Badal RE, Bouchillon SK, Hoban DJ, Hackel MA, Biedenbach DJ, Goff DA: Susceptibility of gram-negative aerobic bacilli from intra-abdominal pathogens to antimicrobial agents collected in the United States during 2011. J Infect 2014, 68:71-76.

17. Karlowsky JA, Adam HJ, Baxter MR, Lagace-Wiens PR, Walkty AJ, Hoban DJ, Zhanel GG: In vitro activity of ceftaroline-avibactam against gram-negative and gram-positive pathogens isolated from patients in Canadian hospitals from 2010 to 2012: results from the CANWARD surveillance study. Antimicrob Agents Chemother 2013, 57:5600-5611.

18. Sader HS, Farrell DJ, Flamm RK, Jones RN: Antimicrobial susceptibility of gram-negative organisms isolated from patients hospitalized in intensive care units in United States and European hospitals (2009-2011). Diagn Microbiol Infect Dis 2014, 78:443-448.

19. Clinical and Laboratory Standards Institute: Performance Standards for Antimicrobial Susceptibility Testing. Twenty-Fourth Informational Supplement. M100-S24. Clinical and Laboratory Standards Institute. Wayne, PA: CLSI; 2014. Document M100-S24.

20. Villar HE, Danel F, Livermore DM: Permeability to carbapenems of Proteus mirabilis mutants selected for resistance to imipenem or other $\beta$-lactams. J Antimicrob Chemother 1997, 40:365-370.

21. Jean SS, Hsueh PR, Lee WS, Yu KW, Liao CH, Chang FY, Ko WC, Wu JJ, Chen YH, Chen YS, Liu JW, Lu MC, Liu CY, Lam C, Chen RJ: Carbapenem susceptibilities and non-susceptibility concordance to different carbapenems amongst clinically important gram-negative bacteria isolated from intensive care units in Taiwan: results from the Surveillance of Multicentre Antimicrobial Resistance in Taiwan (SMART) in 2009. Int Antimicrob Agents 2013, 41:457-462.

22. Lau SM, Peng MY, Chang FY: Resistance rates to commonly used antimicrobials among pathogens of both bacteremic and non-bacteremic community-acquired urinary tract infection. J Microbiol Immunol Infect 2004, 37:185-191.

23. Kuo SC, Chang SC, Wang HY, Lai JF, Chen PC, Shiau YR, Huang IW, Lauderdale TL: Emergence of extensively drug-resistant Acinetobacter baumannii complex over 10 years: nationwide data from the Taiwan Surveillance of Antimicrobial Resistance (TSAR) program. BMC Infect Dis 2012, 12:200.

24. Clinical and Laboratory Standards Institute: Methods for Dilution Antimicrobial Susceptibility tests for Bacteria That Grow Aerobically; Approved Standard-Ninth Edition. CLSI documents M07-A09. Clinical and Laboratory Standards Institute. Wayne, PA, USA: CLSI; 2012.

25. Clinical and Laboratory Standards Institute: Performance Standards for Antimicrobial Susceptibility Testing. Nineteenth Informational Supplement. M100-S19. Clinical and Laboratory Standards Institute (CLSI). Wayne, PA: CLSI; 2009. Document M100-S19.

26. Monstein HJ, Ostholm-Balkhed A, Nilsson MV, Nilsson M, Dornbusch K, Nilsson LE: Multiplex PCR amplification assay for the detection of bla $a_{\mathrm{SHV}}, b / a_{\mathrm{TEM}}$ and bla $a_{\mathrm{CTX}-\mathrm{M}}$ genes in Enterobacteriaceae. APMIS 2007, 115:1400-1408.

27. Perez-Perez FJ, Hanson ND: Detection of plasmid-mediated AmpC $\beta$-lactamase genes in clinical isolates by using multiplex PCR. J Clin Microbiol 2002, 40:2153-2162.

28. O'Brien TF, Stelling J: Integrated multilevel surveillance of the world's infecting microbes and their resistance to antimicrobial agents. Clin Microbiol Rev 2011, 24:281-295.

29. Castanheira M, Farrell SE, Krause KM, Jones RN, Sader HS: Contemporary diversity of $\beta$-lactamases among Enterobacteriaceae in the nine U.S. Census regions and ceftazidime-avibactam activity tested against isolates producing the most prevalent $\beta$-lactamase groups. Antimicrob Agents Chemother 2014, 58:833-838.

30. Su CH, Wang JT, Hsiung CA, Chien LJ, Chi CL, Yu HT, Chang FY, Chang SC: Increase of carbapenem-resistant Acinetobacter baumannii infection in acute care hospitals in Taiwan: association with hospital antimicrobial usage. PLoS One 2012, 7:e37788. 
31. European Committee on Antimicrobial Susceptibility Testing: MIC distributions. 2012. http://www.eucast.org/mic_distributions/ [Accessed 01.04.14].

32. Centers for Diseases Control and Prevention: The CDC guidance for control of CRE-2012 CRE toolkit. Available from http://www.cdc.gov/hai/pdfs/cre/ CRE-guidance-508.pdf.

33. Dudley MN, Ambrose PG, Bhavnani SM, Craig WA, Ferraro MJ, Jones RN: Background and rationale for revised clinical and laboratory standards institute interpretive criteria (breakpoints) for Enterobacteriaceae and Pseudomonas aeruginosa: I. Cephalosporins and aztreonam. Clin Infect Dis 2013, 56:1301-1309.

34. Hawser SP, Badal RE, Bouchillon SK, Hoban DJ, Hsueh PR: Comparison of CLSI 2009, CLSI 2010 and EUCAST cephalosporin clinical breakpoints in recent clinical isolates of Escherichia coli, Klebsiella pneumoniae and Klebsiella oxytoca from the SMART global surveillance study. Int J Antimicrob Agents 2010, 36:293-294.

35. Tan TY, Ng LS, He J, Chen DM: Improved detection of AmpC $\beta$-lactamases in Escherichia coli, Klebsiella pneumoniae, and Proteus mirabilis using new susceptibility breakpoints for third-generation cephalosporins. Diagn Microbiol Infect Dis 2011, 70:423-424.

36. Rossolini GM, D'Andrea MM, Mugnaioli C: The spread of CTX-M-type extended-spectrum $\beta$-lactamases. Clin Microbiol Infect 2008, 14(Suppl 1):33-41.

doi:10.1186/1471-2334-14-486

Cite this article as: Wang et al: Antimicrobial susceptibilities of Proteus mirabilis: a longitudinal nationwide study from the Taiwan surveillance of antimicrobial resistance (TSAR) program. BMC Infectious Diseases 2014 14:486.

\section{Submit your next manuscript to BioMed Central and take full advantage of:}

- Convenient online submission

- Thorough peer review

- No space constraints or color figure charges

- Immediate publication on acceptance

- Inclusion in PubMed, CAS, Scopus and Google Scholar

- Research which is freely available for redistribution 